\title{
Autophagy as a Vital Therapy Target for Renal Cell Carcinoma
}

\author{
Ying-hua $\mathrm{He}^{1 *}$ and Guo Tian $^{2}$ \\ ${ }^{1}$ Department of Clinical Pharmacy, The First Affiliated Hospital, College of Medicine, Zhejiang University, Hangzhou, China, \\ ${ }^{2}$ Hepatobiliary and Pancreatic Intervention Center, The First Affiliated Hospital, College of Medicine, Zhejiang University, \\ Hangzhou, China
}

Autophagy is a process that degrades and recycles superfluous organelles or damaged cellular contents. It has been found to have dual functions in renal cell carcinoma (RCC). Many autophagy-related proteins are regarded as prognostic markers of RCC. Researchers have attempted to explore synthetic and phytochemical drugs for RCC therapy that target autophagy. In this review, we highlight the importance of autophagy in RCC and potential treatments related to autophagy.

Keywords: autophagy, rcc, inhibitor, inducers, PI3K/AKT/mTOR, AMPK/mTOR

\section{BACKGROUND}

\section{OPEN ACCESS}

Edited by:

Norberto Perico,

Istituto di Ricerche Farmacologiche Mario Negri (IRCCS), Italy

Reviewed by:

Xiao-ming Meng, Anhui Medical

University, China

Monica Cortinovis,

Istituto di Ricerche Farmacologiche Mario Negri (IRCCS), Italy

${ }^{*}$ Correspondence: Ying-hua $\mathrm{He}$ hyh735637773@zju.edu.cn

Specialty section: This article was submitted to

Renal Pharmacology, a section of the journal

Frontiers in Pharmacology

Received: 07 December 2019 Accepted: 23 December 2020 Published: 10 February 2021

Citation:

He Yand Tian G (2021) Autophagy as a Vital Therapy Target for Renal Cell Carcinoma. Front. Pharmacol. 11:518225. doi: 10.3389/fphar.2020.518225
Renal cell carcinoma (RCC) is the 14th most common type of cancer globally, which comprises about 80 to $90 \%$ of malignant renal tumors (Hancock and Georgiades, 2016). Its incidence has been increasing at a rate of about $2 \%$ annually, with more than 200,000 new cases worldwide per year (Siegel et al., 2019). Despite advances in surgical techniques, the overall 5-year survival rate of patients in early-stage RCC is approximately $93 \%$, whereas that of patients with metastatic RCC is approximately12\% (Siegel et al., 2017; Attalla et al., 2020). Therefore, it is imperative to develop novel targets against RCC. Nevertheless, many studies have recently reported intimate relationships between autophagy and RCC, which may offer new options for the treatment of RCC.

Based on the different transport modes of intracellular components to the lysosome, autophagy could be categorized into three subtypes: macroautophagy, microautophagy, and chaperonemediated autophagy (CMA) (Jacob et al., 2017). Macroautophagy is the most common subtype. The process of autophagy could be divided into four stages: initiation, elongation and completion, maturation, and fusion or degradation (Limpert et al., 2018; Cao and Bai, 2019). These ultrastructural studies indicate that at the autophagosome initiation stage, inhibition of mTOR induced the formation of ATG1/Unc-51-like kinases (ULK) complex (ULK1/2, ATG13, FIP200, and ATG101). This complex activates phosphatidylinositol 3-phosphate (PI3P) production through class III phosphatidylinositol 3-kinase complex (VPS34, ATG14 L, VPS15, and Beclin1). The production of PI3P recruits certain effectors, including proteins like double FYVE-containing protein 1 (DFCP1), and WD-repeat protein interacting with phosphoinositides (WIPIs) to form omegasomes (nucleation sites) (Mizushima et al., 2011; Devereaux et al., 2013). Possible sources of the autophagosomal membrane may include the endoplasmic reticulum (ER), mitochondria, Golgi apparatus, plasma membrane, and recycling endosomes (Karanasios et al., 2016). At the elongation and completion stage, the vesicle is further extended, curved, and closed to form the autophagosome, which is a double-layer membrane structure with approximately 500 to $1,500 \mathrm{~nm}$ diameter (Hale et al., 2013). This process requires two pivotal ubiquitin-like conjugation systems such as the ATG5-ATG12 complex, which conjugates with ATG16L1, and the microtubule-associated protein 1 light chain 3 (MAP1LC3, commonly called LC3), which conjugates with the lipid phosphatidylethanolamine (PE), commonly referred to as membrane-bound LC3-II (Klionsky 
and Schulman, 2014). During autophagy, LC3-I (LC3 precursor) undergoes cleavage and lipidation to form LC3-II, which is a crucial component of autophagosomes (Ogata et al., 2006). Next, LC3-II is recruited to the two sides of the autophagosome and acts as a receptor, which interacts with adaptor proteins like p62/ SQSTM1 (Hirano et al., 2016). At the maturation stage, the autophagosome fuses with the lysosome to form an autolysosome (Noda and Inagaki, 2015). Lastly, at the fusion or degradation stage, the intracellular components are degraded and released into the cytosol (Tanida et al., 2005).

It is worth noting that research on the relationship between autophagy and RCC has become a subject of interest. Previous studies have established that autophagy-related proteins could act as promising prognostic biomarkers for the treatment of RCC (Q. Deng et al., 2018; Guo et al., 2019; Nishikawa et al., 2015). Bray et al. found that human RCCs have high basal autophagy which is required for survival to mTOR inhibition (Bray et al., 2012). It is attractive for autophagy inducers or inhibitors for the treatment of RCC. Therefore, this study aims to explore the molecular mechanisms of autophagy, focusing on the systematic analysis of autophagy in RCC.

\section{Autophagy-Related Proteins in RCC}

Previously it was observed that Beclin1 is involved in assembling Beclin 1-Vps34-Vps15 core complexes and inducing autophagy, particularly under unfavorable conditions (R. Kang et al., 2011). Additionally, Bcl-2 inhibited autophagy through binding to Beclin1 (Grimaldi et al., 2015). Moreover, univariate and multivariate analysis showed that the expression level of Beclin1 appeared to be negatively correlated to the recurrencefree survival (RFS) in 100 patients with non-metastatic renal clear cell carcinoma (ccRCC) (Nishikawa et al., 2015). The high expression of Beclin 1 was also identified in tissues and cells of RCC (A498 and ACHN cell lines) (Guo et al., 2019).

The increased conversion of LC3-I to LC3-II is considered a marker for the initiation of autophagy because of its aggregation and localization on autophagosomes (Ogata et al., 2006). This research has revealed that cell mobility in ccRCC, A498, and ACHN cell lines is promoted by the up-regulated expression of LC3 (Guo et al., 2019). Two reports showed that promoting autophagy-related apoptosis resulted in down-regulation of LC3II levels in RCC tissues and cells (Grimaldi et al., 2015; M. L.; Li et al., 2018). By contrast, Wang et al. found that LC3-II expression levels in RCC cell lines (786-O, 769-P, OS-RC-2, ACHN cells) were lower than those in a control cell line (HK-2 cell) (Wang et al., 2018).

Importantly, low expression of ATGs that is related to the process of autophagy nucleation, predicts poor prognosis in RCC (X. D. Liu et al., 2015a; Yu et al., 2018). Liu et al. found that most ccRCCs harbor allelic loss and/or mutation of ATG7. The efficient ATG7 down-regulation suppressing autophagy in RCC cell lines was evidenced by the down-regulation of LC3II (Q. Deng et al., 2018; Wang et al., 2018). Studies have confirmed that the expression levels of ATG5-ATG12 conjugates is positively correlated with LC3-II aggregation and Beclin1 expression in RCC (M. L. Li et al., 2018; Turcotte et al., 2008).
P62/SQSTM1, a classical macroautophagy substrate, binds directly to LC3 to degrade ubiquitinated protein (Pankiv et al., 2007). Autophagy decreased P62 levels in RCC (Wu et al., 2015). Furthermore, p62 amplification on chromosome $5 \mathrm{q}$ was linked to renal cancer tumorigenesis (Mathew et al., 2009).

\section{Autophagy-Related Tumor Suppressor Genes in RCC}

Research has shown that genetic dysregulation of autophagy is a key characteristic of different subtypes of RCC (X. D. Liu et al., 2015b). The Von Hippel-Lindau (VHL) tumor suppressor is lost on the short arm of chromosome 3 in the majority of ccRCC (80\%). It is well known that loss of VHL leads to induction of the hypoxia inducible factor (HIF), which in turn promotes tumor growth (Gossage and Eisen, 2010). Mikhaylova et al. demonstrated that VHL regulated autophagy in ccRCC. They found that inhibition of autophagy by knockdown of ATG5 resulted in the massive death of VHL(-) cells as compared to VHL(+) cells, indicating that VHL(-) cells could be more dependent on autophagy and therefore more sensitive to inhibition (Mikhaylova et al., 2012). VHL(-) cells also facilitate, in a HIF-independent manner, intracellular nutrients by activation of LC3B-mediated autophagy, which are necessary for tumor growth. Recently, it was reported that VHL mutation in RCC cells induced autophagy by up-regulating the inositol 1,4,5trisphosphate receptor, type 1 (ITPR1) (Messai et al., 2014).

Folliculin (FLCN) is a tumor suppressor gene that is deficient in Birt-Hogg-Dube syndrome (BHD), a disorder that features renal carcinoma of multiple histological types including hybrid oncocytic RCC, chromophobe RCC, oncocytoma, multiple and bilateral clear cell RCC (Verine et al., 2010). FLCN protein expression is reduced in ccRCC following loss of VHL, and it predicts poor prognosis of RCC (Schmidt et al., 2005). Previously, Bastola et al. illustrated that FLCN promoted autophagy processes by activating the mTORC1 activity in ccRCC cell lines. This indicates that FLCN contributes to the tumorsuppressing effect of VHL (Bastola et al., 2013). Subsequently, Zhang et al. found that paclitaxel-induced autophagy prevented the apoptosis of FLCN-deficient RCC cells. Thus, paclitaxel combined with autophagy inhibitors might be an effective treatment for FLCN-deficient RCC (Zhang et al., 2013).

P53 is up-regulated in tumor tissues, where it may inhibit tumor progression via autophagy. Recent studies have shown that p53 has dual effects in autophagy depending on the subcellular localization. Nuclear p53 facilitates autophagy whereas cytoplasmic p53 inhibits autophagy (Tang et al., 2015; White, 2016). Autophagy also regulates p53. Previously, Kang and $\mathrm{Ku}$ et al. found that, in RCC cell lines, transglutaminase 2 (TGase 2), an enzyme regulating covalent crosslinking between protein glutamine and lysine residues, cross-linked p53 into the autophagosome, thereby down-regulating p53 (J. H. Kang et al., 2016; Ku et al., 2013). After the treatment of chloroquine (CQ) and MG132, the binding of p53 with TGase 2 and p62 was potentiated (Ku et al., 2013).

A DNA senor in the cytoplasm that could bind to doublestranded DNA (dsDNA) to cause inflammatory cell death, called 
pyroptosis (Rathinam et al., 2010), is absent in the tumor suppression melanoma 2 (AIM2). AIM2 enhances the expression of autophagy-related genes in vitro and in vivo. The low expression levels of AIM2 in RCC reduce autophagy and promote tumorigenesis (Chai et al., 2018).

\section{Autophagy-Related IncRNAs in RCC}

Long non-coding RNAs (lncRNAs) are a class of RNAs that are more than 200 nucleotides in length and could not be translated into proteins (Hong et al., 2017). The silencing of a lncRNA, known as the HOXA transcript at the distal tip (HOTTIP), can induce autophagy by increasing a multitude of autophagy-related genes including Beclin1, LC3B, and LAMP2 through the PI3K/ Akt/Atg13 signaling pathway (Su et al., 2019). A recent study by Shao et al. suggested that the IncRNA, a Secretory Carrier Membrane Protein 1 (SCAMP1), inhibited RCC tumorigenesis through activating autophagy in RCC cells (Shao et al., 2019).

\section{Autophagy-Related Signaling Pathways in RCC $\mathrm{PI}$ IK/Akt/mTOR Pathway}

Even though there are two forms of mTOR in mammals, most studies only pay attention to mTOR1 because of its sensitivity to rapamycin. The activation of the PI3K/Akt pathway enhances cell survival by inhibiting apoptosis and promoting cell cycle progression by activating $\mathrm{mTORC1}$, thereby inhibiting autophagy (Ren et al., 2016; Deng et al., 2018). For example, Hongyan et al. found that NVP-BEZ235, a novel dual PI3K/ mTOR inhibitor, induced cell apoptosis and autophagy in RCC (H. Li et al., 2013). Furthermore, Antonaci et al. indicated that dimethyl sulfide (DMS) induced autophagy in Caki-1 cells through $\mathrm{PI} 3 \mathrm{~K} / \mathrm{AKT} / \mathrm{mTOR} / \mathrm{p} 70 \mathrm{~S} 6 \mathrm{~K}$ pathways (Antonaci et al., 2019). Sunitinib blocking the Akt/mTOR/p70S6K pathway has resulted in autophagy activation in vivo and in vitro (M. L. Li et al., 2018). Deng et al. also reported that sinomenine induced cell apoptosis and autophagy in ACHN cells by making the PI3K/ Akt/mTOR signaling pathway inactive (F. Deng et al., 2018). These results suggest that the $\mathrm{PI} 3 \mathrm{~K} / \mathrm{Akt} / \mathrm{mTOR}$ signaling pathway and autophagy are important avenues for the prevention and treatment of RCC.

\section{AMPK/mTOR Pathway}

During metabolic stress, high AMP/ATP ratio adenosine activates monophosphate-activated protein kinase (AMPK). AMPK then inhibits mTOR by activating the TSC1/TSC2 protein heterodimer, which results in autophagy activation (Kim et al., 2011). In addition, the AMPK pathway modulates autophagy via an alternative mechanism, in which AMPK stimulates ULK1 and facilitates autophagy due to the phosphorylation of Ser317 and Ser777 (Kim et al., 2011). A recent study found that silibinin repressed the phosphorylation levels of mTOR, increasing the level of AMPK, and markedly promoting the expression of the autophagy marker LC3-II. Moreover, the effects of silibinin on mTOR and autophagy were reversed by compound $\mathrm{C}$, a pharmacological inhibitor of AMPK (F. Li et al., 2015). It has also been reported that curcumin has activated autophagy in 786- $\mathrm{O}$ and $\mathrm{ACHN}(5$ and $20 \mu \mathrm{M}$ ) via the AMPK signaling pathway (Q. Deng et al., 2018). Resveratrol also induced autophagy of Ketr-3 cells by activating p53/AMPK/ mTOR leading to apoptosis of RCC cells (Q. Liu et al., 2018). Among these, the AMPK/mTOR pathway is the primary regulator of autophagy in RCC.

\section{The Dual Roles of Autophagy in RCC}

Although evidence that autophagy regulates both cell survival and death is available, it is not clear why autophagy has dual effects.

Activation of autophagy suppresses tumors by eliminating dysfunctional proteins and damaged cellular organelles and maintaining host defenses (Liang and Jung, 2010). Reduced and aberrant expression of autophagy genes and proteins may affect various aspects of RCC pathology. It has been reported that the cytoprotective enzyme heme oxygenase-1(HO-1) downregulated autophagy-related proteins Beclin-1 and LC3B-II in renal cancer cells (Banerjee et al., 2012). Wang et al. found that activation of autophagy by Atg7 and LC3-II overexpression suppressed cell proliferation in 786-O, 769-P, OS-RC-2, ACHN human RCC cell lines in vivo and in vitro (Wang et al., 2018).

On the other hand, autophagy protects some tumor cells against low-oxygen conditions and nutrient deprivation, which are the main characteristics of tumor microenvironments (Katheder et al., 2017). In VHL-deficient RCC cells, EPAS1, a type of hypoxia-inducible factor, is not degraded but accumulated targeting ITPR1. Meanwhile, ITPR1 regulates sensing a yet undefined signal derived from NK cells to activate autophagy. Activation of autophagy in RCC cells results in the degradation of NK-derived granzyme B (GZMB) which compromises the NKmediated killing of tumor cells (Messai et al., 2014; Messai et al., 2015). It has also been proven that ITPR1 regulates the NKmediated killing of RCC cells through the activation of autophagy (Messai et al., 2015). Researchers have reported that various chemotherapeutic drugs for RCC may increase the autophagic flux of RCC cells, and chemotherapeutic drugs combined with autophagy inhibitors may be more effective in controlling RCC progression. For example, temsirolimus (TEM), an mTOR inhibitor, showed good performance on advanced RCC (Voss et al., 2011). However, the effect of TEM is transient in most patients. A study showed that autophagy had protective mechanisms in the regulation of resistance to TEM in RCC (Singla and Bhattacharyya, 2017; Chow et al., 2020). Moreover, the inhibition of autophagy with CQ increased the risk of TEM-induced cell death (Singla and Bhattacharyya, 2017). The Kringle 1 domain of human hepatocyte growth factor (HGFK1) was found to enhance the anti-tumor activities of sorafenib and reverse resistance to this drug in RCC via the inhibition of autophagy (Gao et al., 2019). Recently, a phase I/II trial in patients with RCC showed that the autophagy inhibition achieved by hydroxychloroquine enhanced the anti-tumor effects of mTOR inhibitors (Haas et al., 2019). The PI3K/mTOR dual inhibitor NVP-BEZ235 (50, 100 or 500 and 1,000 nM) induced 786-0 cells apoptosis and autophagy by increasing LC3-II and decreasing p62. Autophagy inhibitors significantly potentiated the anticancer effect of NVP-BEZ235 (H. Li et al., 2013). 
TABLE 1 | Modulate autophagy compounds.

\begin{tabular}{|c|c|c|c|c|c|}
\hline Compound & In vitro/in vivo & Target & $\begin{array}{l}\text { Regulate } \\
\text { autophagy }\end{array}$ & Biological role & Refernces \\
\hline STF-62247 & in vitro and in vivo & LC3-II & Induce & Cell death $(+)$ & Anbalagan et al. (2012) \\
\hline Ubenimex & in vitro & LC3B & Induce & Cell death $(+)$ & Liu et al. (2015) \\
\hline $\begin{array}{l}\text { (R)-goniothalamin and (S)- } \\
\text { goniothalamin }\end{array}$ & in vitro & LC3B & Induce & Cell death $(+)$ & de Fatima et al. (2008) \\
\hline Silibinin & in vitro and in vivo & LC3-II & Induce & Metastasis (-) & F. Li et al. (2015) \\
\hline Resveratrol & in vitro & ATG5, ATG7 & Induce & Apoptosis (+) & Q. Liu et al. (2018) \\
\hline Sinomenine & in vitro & $\begin{array}{c}\text { Beclin1, LC3-II/LC3- } \\
\text { I, p62 }\end{array}$ & Induce & Apoptosis (+) & F. Deng et al. (2018) \\
\hline Rasfonin & in vitro & LC3-II, p62 & Induce & $\begin{array}{l}\text { Apoptosis (+), } \\
\text { necroptosis (+) }\end{array}$ & Sun et al. (2016) \\
\hline Chloroquine & in vitro & Deacidifying the lysosome & Inhibit & Apoptosis (+) & Grimaldi et al. (2015) \\
\hline 3-Methyladenine & in vitro & LC3-II & Inhibit & Apoptosis (+) & Zhang et al. (2013) \\
\hline Bafilomycin A1 & in vitro & Deacidifying the lysosome & Inhibit & Apoptosis (+) & Zhang et al. (2013) \\
\hline
\end{tabular}

$(+)=$ increased, $(-)=$ decreased.

\section{Autophagy and Therapy in RCC Autophagy Inducers as Treatments for RCC}

Studies have reported that the activation of autophagy may exert therapeutic effects against RCC, some of which are shown in Table 1. Mutations and/or inactivation of the VHL tumor suppressor gene exist in most RCC and are relevant to poor prognosis. Turcotte et al. (Turcotte et al., 2008) designed a compound, STF-62247, which selectively targeted VHLdeficient cells in vitro and in vivo. The compound enhanced autophagy by influencing Golgi trafficking and PI3K passage in VHL-deficient cells. VHL is a significant negative regulator of HIF-a. They found that STF-62247 induced autophagy in a HIFindependent manner. The VHL-deficient RCC was more sensitive to STF-62247 compared to cells with wild-type VHL, establishing a synthetic lethal situation due to the combining drug treatment and VHL deficiency. In addition, STF-62247 increased the radiosensitivity of VHL-deficient RCC cells and 786-O cells by inducing autophagy (Anbalagan et al., 2012). Ubenimex induced RCC cell death by upregulating autophagy, as evidenced by increased LC3B (S. Liu et al, 2015C). (R)goniothalamin and (S)-goniothalamin, a pair of styryllactone enantiomer extracted from plants of the genus Goniothalamus, induced the death of human kidney cancer cells (786-0) primarily by enhancing the expression of LC3B (de Fatima et al., 2008). Silibinin is a flavonoid derived from the seeds of milk thistle. The study showed that autophagy induction by silibinin positively contributing to its anti-metastatic capacity in human RCC cells (ACHN and 786-O) by increasing the expression of LC3-II and enrichment of autophagolysosome vacuoles via the AMPK/ mTOR pathway (F. Li et al., 2015). Resveratrol induced Ketr-3 cells apoptosis by triggering ATG5 and ATG7 expression through p53-mediated AMPK/mTOR signaling (Q. Liu et al., 2018). In ACHN cell lines, sinomenine, an isoquinoline extracted from Sinomenium acutum, inhibited RCC progression by inducing autophagy via Beclin1 and LC3-II/LC3-I up-regulation and p62 down-regulation (F. Deng et al., 2018). Rasfonin (A304), a product of Talaromyces $s p$, activates the death of RCC cells by inducing autophagy, and this effect could be suppressed by the Akt inhibitor (Lu et al., 2015; Sun et al., 2016).

\section{Autophagy Inhibitors for the Treatment of RCC}

As discussed in previous sections, autophagy may be a survival mechanism in most cellular contexts as it prevents or delays cancer cell death (Altman and Rathmell, 2012). In cancer cells exposed to stress stimuli such as hypoxia, nutrient deficiency, and chemotherapy, autophagy is activated as a protective mechanism to maintain the survival of cancer cells (Mathew and White, 2011). It has been found that autophagy induced by heteronemin partially antagonized cytotoxicity and apoptotic signaling in human renal carcinoma A498 cells (Wu et al., 2015). Chauhan et al. demonstrated that autophagy inhibitors enhanced apoptosis in A498 cells (Chauhan et al., 2017). Therefore, autophagy is considered a novel therapeutic target (Table 1).

Grimaldi et al. identified that chloroquine (CQ) improved the efficacy of everolimus and sunitinib by down-regulating autophagy in RCC cells (Grimaldi et al., 2015; M. L.; Li et al., 2018). Similar to CQ, hydroxychloroquine (HCQ) inhibits autophagy via deacidifying the lysosome to block its fusion with autophagosome (Levy et al., 1997). Four clinical trials of autophagy are currently ongoing to test the performance single HCQ or its combination with other drugs on RCC (Levy et al., 1997). Elsewhere, it was found that paclitaxel-activated apoptosis induced by the inhibition of autophagy with 3-Methyladenine (3MA) and bafilomycin significantly enhanced in FLCN-deficient RCC cells (Zhang et al., 2013). Interestingly, bafilomycin A1 was found to block autophagy by inhibiting the fusion of autophagosome and lysosome. Hence, a combination of autophagy inhibitors and other therapies may effectively control RCC.

\section{CONCLUSION}

Autophagy is modulated by multiple intracellular processes in varied stressful conditions, such as during organelle dysfunction, nutrient deprivation, and anticancer therapy. The data reviewed 


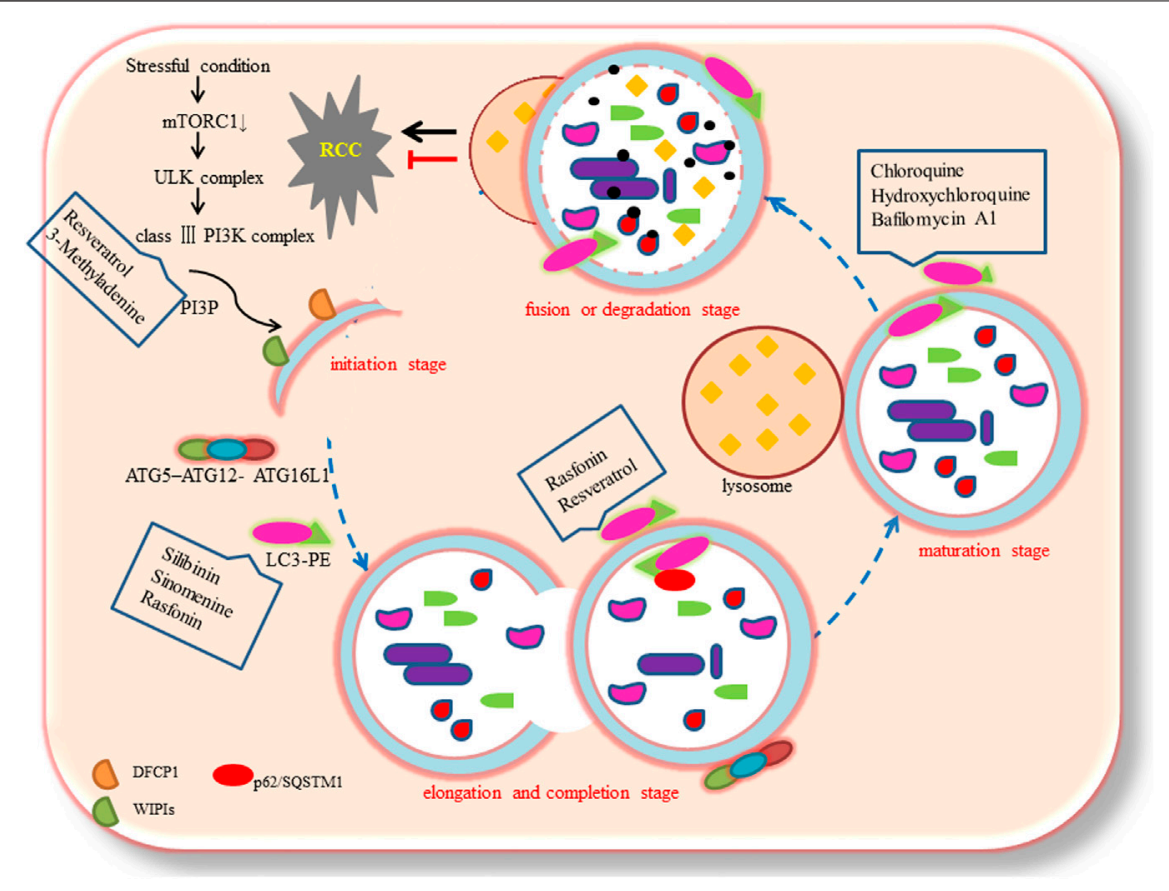

FIGURE 1 | The process of autophagy.

here show that autophagy has a dual role in the initiation, progression, treatment, and drug resistance of RCC. In general, autophagy suppresses tumor development by eliminating oxidative stress, maintaining genomic stability, and reducing dysfunctional proteins in RCC. However, chemotherapies targeting RCC activate autophagy inducing drug tolerance and hence promote tumor progression. This indicates that drugs that restrain autophagy could be effective treatments for RCC. In other words, chemotherapies combined with autophagy inhibitors may be more effective, especially for chemotherapy-resistant RCC. Studies have documented that autophagy regulators may regulate the $\mathrm{AMPK} / \mathrm{mTOR}$ and $\mathrm{PI} 3 \mathrm{~K} / \mathrm{Akt} / \mathrm{mTOR}$ signaling

\section{REFERENCES}

Altman, B. J., and Rathmell, J. C. (2012). Metabolic stress in autophagy and cell death pathways. Cold Spring Harb. Perspect. Biol. 4 (9), a008763. doi:10.1101/ cshperspect.a008763

Anbalagan, S., Pires, I. M., Blick, C., Hill, M. A., Ferguson, D. J., Chan, D. A., et al. (2012). Radiosensitization of renal cell carcinoma in vitro through the induction of autophagy. Radiother. Oncol. 103 (3), 388-393. doi:10.1016/j.radonc.2012.04.001

Antonaci, G., Cossa, L. G., Muscella, A., Vetrugno, C., De Pascali, S. A., Fanizzi, F. P., et al. (2019). [Pt(O,O'-acac)(gamma-acac)(DMS)] induces autophagy in Caki-1 renal cancer cells. Biomolecules. 9 (3), 92. doi:10.3390/ biom 9030092

Attalla, K., Weng, S., Voss, M. H., and Hakimi, A. A. (2020). Epidemiology, risk assessment, and biomarkers for patients with advanced renal cell carcinoma. Urol. Clin. 47 (3), 293-303. doi:10.1016/j.ucl.2020.04.002

Banerjee, P., Basu, A., Wegiel, B., Otterbein, L. E., Mizumura, K., Gasser, M., et al. (2012). Heme oxygenase-1 promotes survival of renal cancer cells through modulation of apoptosis- and autophagy-regulating molecules. J. Biol. Chem. 287 (38), 32113-32123. doi:10.1074/jbc.M112.393140 pathways to confer therapeutic effects in RCC. Further research is needed to reveal the clinical significance of autophagy inhibitors and activators in RCC.

How to control and exploit autophagy for diagnostics and treatment in RCC needs further discussion. We believe that further research on autophagy will lead to the design of important therapeutic strategies for the treatment of RCC.

\section{AUTHOR CONTRIBUTIONS}

$\mathrm{YH}$ wrote the paper. GT reviewed the paper.

Bastola, P., Stratton, Y., Kellner, E., Mikhaylova, O., Yi, Y., Sartor, M. A., et al. (2013). Folliculin contributes to VHL tumor suppressing activity in renal cancer through regulation of autophagy. PloS One. 8 (7), e70030. doi:10.1371/journal. pone.0070030

Bray, K., Mathew, R., Lau, A., Kamphorst, J. J., Fan, J., Chen, J., et al. (2012). Autophagy suppresses RIP kinase-dependent necrosis enabling survival to mTOR inhibition. PloS One. 7 (7), e41831. doi:10.1371/journal.pone.0041831

Cao, Q., and Bai, P. (2019). Role of autophagy in renal cancer. J. Canc. 10 (11), 2501-2509. doi:10.7150/jca.29285

Chai, D., Shan, H., Wang, G., Li, H., Fang, L., Song, J., et al. (2018). AIM2 is a potential therapeutic target in human renal carcinoma and suppresses its invasion and metastasis via enhancing autophagy induction. Exp. Cell Res. 370 (2), 561-570. doi:10.1016/j.yexcr.2018.07.021

Chauhan, A., Ojha, R., Semwal, D. K., Mishra, S. P., and Semwal, R. B. (2017). Combined treatment with CCI779 and SB203580 induces cellular senescence in renal cell carcinoma cell line via p53 pathway. Anticanc. Agents Med. Chem. 17 (11), 1545-1554. doi:10.2174/ 1871520617666170213111002

Chow, P. M., Liu, S. H., Chang, Y. W., Kuo, K. L., Lin, W. C., and Huang, K. H. (2020). The covalent CDK7 inhibitor THZ1 enhances temsirolimus-induced 
cytotoxicity via autophagy suppression in human renal cell carcinoma. Canc. Lett. 471, 27-37. doi:10.1016/j.canlet.2019.12.005

de Fatima, A., Zambuzzi, W. F., Modolo, L. V., Tarsitano, C. A., Gadelha, F. R., Hyslop, S., et al. (2008). Cytotoxicity of goniothalamin enantiomers in renal cancer cells: involvement of nitric oxide, apoptosis and autophagy. Chem. Biol. Interact. 176 (2-3), 143-150. doi:10.1016/j.cbi.2008.08.003

Deng, F., Ma, Y. X., Liang, L., Zhang, P., and Feng, J. (2018). The pro-apoptosis effect of sinomenine in renal carcinoma via inducing autophagy through inactivating PI3K/AKT/mTOR pathway. Biomed. Pharmacother. 97, 1269-1274. doi:10.1016/j.biopha.2017.11.064

Deng, Q., Liang, L., Liu, Q., Duan, W., Jiang, Y., and Zhang, L. (2018). Autophagy is a major mechanism for the dual effects of curcumin on renal cell carcinoma cells. Eur. J. Pharmacol. 826, 24-30. doi:10.1016/j. ejphar.2018.02.038

Devereaux, K., Dall'Armi, C., Alcazar-Roman, A., Ogasawara, Y., Zhou, X., Wang, F., et al. (2013). Regulation of mammalian autophagy by class II and III PI 3kinases through PI3P synthesis. PloS One. 8 (10), e76405. doi:10.1371/journal. pone.0076405

Gao, X., Jiang, P., Zhang, Q., Liu, Q., Jiang, S., Liu, L., et al. (2019). Peglated-H1/ pHGFK1 nanoparticles enhance anti-tumor effects of sorafenib by inhibition of drug-induced autophagy and stemness in renal cell carcinoma. J. Exp. Clin. Canc. Res. 38 (1), 362. doi:10.1186/s13046-019-1348-z

Gossage, L., and Eisen, T. (2010). Alterations in VHL as potential biomarkers in renal-cell carcinoma. Nat. Rev. Clin. Oncol. 7 (5), 277-288. doi:10.1038/ nrclinonc. 2010.42

Grimaldi, A., Santini, D., Zappavigna, S., Lombardi, A., Misso, G., Boccellino, M., et al. (2015). Antagonistic effects of chloroquine on autophagy occurrence potentiate the anticancer effects of everolimus on renal cancer cells. Canc. Biol. Ther. 16 (4), 567-579. doi:10.1080/15384047.2015.1018494

Guo, Y., Zhang, H. C., Xue, S., and Zheng, J. H. (2019). Receptors for advanced glycation end products is associated with autophagy in the clear cell renal cell carcinoma. J. Canc. Res. Therapeut. 15 (2), 317-323. doi:10.4103/jcrt.JCRT_180_18

Haas, N. B., Appleman, L. J., Stein, M., Redlinger, M., Wilks, M., Xu, X., et al. (2019). Autophagy inhibition to augment mTOR inhibition: a phase I/II trial of everolimus and hydroxychloroquine in patients with previously treated renal cell carcinoma. Clin. Canc. Res. 25 (7), 2080-2087. doi:10.1158/1078-0432. CCR-18-2204

Hale, A. N., Ledbetter, D. J., Gawriluk, T. R., and Rucker, E. B., 3rd (2013). Autophagy: regulation and role in development. Autophagy. 9 (7), 951-972. doi:10.4161/auto.24273

Hancock, S. B., and Georgiades, C. S. (2016). Kidney cancer. Cancer J. 22 (6), 387-392. doi:10.1097/PPO.0000000000000225

Hirano, S., Uemura, T., Annoh, H., Fujita, N., Waguri, S., Itoh, T., et al. (2016). Differing susceptibility to autophagic degradation of two LC3-binding proteins: SQSTM1/p62 and TBC1D25/OATL1. Autophagy. 12 (2), 312-326. doi:10.1080/ 15548627.2015 .1124223

Hong, Q., Li, O., Zheng, W., Xiao, W. Z., Zhang, L., Wu, D., et al. (2017). LncRNA HOTAIR regulates HIF-1alpha/AXL signaling through inhibition of miR-217 in renal cell carcinoma. Cell Death Dis. 8 (5), e2772. doi:10.1038/cddis.2017.181

Jacob, J. A., Salmani, J. M. M., Jiang, Z., Feng, L., Song, J., Jia, X., et al. (2017). Autophagy: an overview and its roles in cancer and obesity. Clin. Chim. Acta. 468, 85-89. doi:10.1016/j.cca.2017.01.028

Kang, J. H., Lee, J. S., Hong, D., Lee, S. H., Kim, N., Lee, W. K., et al. (2016). Renal cell carcinoma escapes death by p53 depletion through transglutaminase 2chaperoned autophagy. Cell Death Dis. 7, e2163. doi:10.1038/cddis.2016.14

Kang, R., Zeh, H. J., Lotze, M. T., and Tang, D. (2011). The Beclin 1 network regulates autophagy and apoptosis. Cell Death Differ. 18 (4), 571-580. doi:10. 1038/cdd.2010.191

Karanasios, E., Walker, S. A., Okkenhaug, H., Manifava, M., Hummel, E., Zimmermann, H., et al. (2016). Autophagy initiation by ULK complex assembly on ER tubulovesicular regions marked by ATG9 vesicles. Nat. Commun. 7, 12420. doi:10.1038/ncomms 12420

Katheder, N. S., Khezri, R., O’Farrell, F., Schultz, S. W., Jain, A., Rahman, M. M., et al. (2017). Microenvironmental autophagy promotes tumour growth. Nature. 541 (7637), 417-420. doi:10.1038/nature20815

Kim, J., Kundu, M., Viollet, B., and Guan, K. L. (2011). AMPK and mTOR regulate autophagy through direct phosphorylation of Ulk1. Nat. Cell Biol. 13 (2), 132-141. doi:10.1038/ncb2152
Klionsky, D. J., and Schulman, B. A. (2014). Dynamic regulation of macroautophagy by distinctive ubiquitin-like proteins. Nat. Struct. Mol. Biol. 21 (4), 336-345. doi:10.1038/nsmb.2787

Ku, B. M., Kim, D. S., Kim, K. H., Yoo, B. C., Kim, S. H., Gong, Y. D., et al. (2013). Transglutaminase 2 inhibition found to induce p53 mediated apoptosis in renal cell carcinoma. Faseb. J. 27 (9), 3487-3495. doi:10.1096/fj.12-224220

Levy, G. D., Munz, S. J., Paschal, J., Cohen, H. B., Pince, K. J., and Peterson, T. (1997). Incidence of hydroxychloroquine retinopathy in 1,207 patients in a large multicenter outpatient practice. Arthritis Rheum. 40 (8), 1482-1486. doi:10.1002/art.1780400817

Li, F., Ma, Z., Guan, Z., Chen, Y., Wu, K., Guo, P., et al. (2015). Autophagy induction by silibinin positively contributes to its anti-metastatic capacity via AMPK/mTOR pathway in renal cell carcinoma. Int. J. Mol. Sci. 16 (4), 8415-8429. doi:10.3390/ijms16048415

Li, H., Jin, X., Zhang, Z., Xing, Y., and Kong, X. (2013). Inhibition of autophagy enhances apoptosis induced by the PI3K/AKT/mTor inhibitor NVP-BEZ235 in renal cell carcinoma cells. Cell Biochem. Funct. 31 (5), 427-433. doi:10.1002/cbf. 2917

Li, M. L., Xu, Y. Z., Lu, W. J., Li, Y. H., Tan, S. S., Lin, H. J., et al. (2018). Chloroquine potentiates the anticancer effect of sunitinib on renal cell carcinoma by inhibiting autophagy and inducing apoptosis. Oncol. Lett. 15 (3), 2839-2846. doi:10.3892/ol.2017.7635

Liang, C., and Jung, J. U. (2010). Autophagy genes as tumor suppressors. Curr. Opin. Cell Biol. 22 (2), 226-233. doi:10.1016/j.ceb.2009.11.003

Limpert, A. S., Lambert, L. J., Bakas, N. A., Bata, N., Brun, S. N., Shaw, R. J., et al. (2018). Autophagy in cancer: regulation by small molecules. Trends Pharmacol. Sci. 39 (12), 1021-1032. doi:10.1016/j.tips.2018.10.004

Liu, Q., Fang, Q., Ji, S., Han, Z., Cheng, W., and Zhang, H. (2018). Resveratrolmediated apoptosis in renal cell carcinoma via the p53/AMPactivated protein kinase/mammalian target of rapamycin autophagy signaling pathway. Mol. Med. Rep. 17 (1), 502-508. doi:10.3892/mmr.2017.7868

Liu, X. D., Yao, J., Tripathi, D. N., Ding, Z., Xu, Y., Sun, M., et al. (2015a). Autophagy mediates HIF2alpha degradation and suppresses renal tumorigenesis. Oncogene. 34 (19), 2450-2460. doi:10.1038/onc.2014.199

Liu, X. D., Zhu, H., DePavia, A., and Jonasch, E. (2015b). Dysregulation of HIF2alpha and autophagy in renal cell carcinoma. Mol. Cell Oncol. 2 (2), e965643. doi:10.4161/23723548.2014.965643

Liu, S., Xie, F., Wang, H., Liu, Z., Liu, X., Sun, L., et al. (2015c). Ubenimex inhibits cell proliferation, migration and invasion in renal cell carcinoma: the effect is autophagy-associated. Oncol. Rep. 33 (3), 1372-1380. doi:10.3892/or.2014.3693

Lu, Q., Yan, S., Sun, H., Wang, W., Li, Y., Yang, X., et al. (2015). Akt inhibition attenuates rasfonin-induced autophagy and apoptosis through the glycolytic pathway in renal cancer cells. Cell Death Dis. 6, e2005. doi:10.1038/cddis. 2015.344

Mathew, R., Karp, C. M., Beaudoin, B., Vuong, N., Chen, G., Chen, H. Y., et al. (2009). Autophagy suppresses tumorigenesis through elimination of p62. Cell. 137 (6), 1062-1075. doi:10.1016/j.cell.2009.03.048

Mathew, R., and White, E. (2011). Autophagy, stress, and cancer metabolism: what doesn't kill you makes you stronger. Cold Spring Harbor Symp. Quant. Biol. 76, 389-396. doi:10.1101/sqb.2012.76.011015

Messai, Y., Noman, M. Z., Hasmim, M., Janji, B., Tittarelli, A., Boutet, M., et al. (2014). ITPR1 protects renal cancer cells against natural killer cells by inducing autophagy. Cancer Res. 74 (23), 6820-6832. doi:10.1158/0008-5472.CAN-140303

Messai, Y., Noman, M. Z., Janji, B., Hasmim, M., Escudier, B., and Chouaib, S. (2015). The autophagy sensor ITPR1 protects renal carcinoma cells from NKmediated killing. Autophagy 0. doi:10.1080/15548627.2015.1017194

Mikhaylova, O., Stratton, Y., Hall, D., Kellner, E., Ehmer, B., Drew, A. F., et al. (2012). VHL-regulated MiR-204 suppresses tumor growth through inhibition of LC3B-mediated autophagy in renal clear cell carcinoma. Canc. Cell. 21 (4), 532-546. doi:10.1016/j.ccr.2012.02.019

Mizushima, N., Yoshimori, T., and Ohsumi, Y. (2011). The role of Atg proteins in autophagosome formation. Annu. Rev. Cell Dev. Biol. 27, 107-132. doi:10.1146/ annurev-cellbio-092910-154005

Nishikawa, M., Miyake, H., Liu, B., and Fujisawa, M. (2015). Expression pattern of autophagy-related markers in non-metastatic clear cell renal cell carcinoma: association with disease recurrence following radical nephrectomy. J. Canc. Res. Clin. Oncol. 141 (9), 1585-1591. doi:10.1007/s00432-015-1923-4 
Noda, N. N., and Inagaki, F. (2015). Mechanisms of autophagy. Annu. Rev. Biophys. 44, 101-122. doi:10.1146/annurev-biophys-060414-034248

Ogata, M., Hino, S., Saito, A., Morikawa, K., Kondo, S., Kanemoto, S., et al. (2006). Autophagy is activated for cell survival after endoplasmic reticulum stress. Mol. Cell Biol. 26 (24), 9220-9231. doi:10.1128/MCB.01453-06

Pankiv, S., Clausen, T. H., Lamark, T., Brech, A., Bruun, J. A., Outzen, H., et al. (2007). p62/SQSTM1 binds directly to Atg8/LC3 to facilitate degradation of ubiquitinated protein aggregates by autophagy. J. Biol. Chem. 282 (33), 24131-24145. doi:10.1074/jbc.M702824200

Rathinam, V. A., Jiang, Z., Waggoner, S. N., Sharma, S., Cole, L. E., Waggoner, L., et al. (2010). The AIM2 inflammasome is essential for host defense against cytosolic bacteria and DNA viruses. Nat. Immunol. 11 (5), 395-402. doi:10. 1038/ni.1864

Ren, C., Ren, T., Yang, K., Wang, S., Bao, X., Zhang, F., et al. (2016). Inhibition of SOX2 induces cell apoptosis and G1/S arrest in Ewing's sarcoma through the PI3K/Akt pathway. J. Exp. Clin. Canc. Res. 35, 44. doi:10.1186/s13046-0160321-3

Schmidt, L. S., Nickerson, M. L., Warren, M. B., Glenn, G. M., Toro, J. R., Merino, M. J., et al. (2005). Germline BHD-mutation spectrum and phenotype analysis of a large cohort of families with Birt-Hogg-Dube syndrome. Am. J. Hum. Genet. 76 (6), 1023-1033. doi:10.1086/430842

Shao, Q., Wang, Q., and Wang, J. (2019). LncRNA SCAMP1 regulates ZEB1/JUN and autophagy to promote pediatric renal cell carcinoma under oxidative stress via miR-429. Biomed. Pharmacother. 120, 109460. doi:10.1016/j.biopha.2019. 109460

Siegel, R. L., Miller, K. D., and Jemal, A. (2017). Cancer statistics, 2017. CA Canc. J. Clin. 67 (1), 7-30. doi:10.3322/caac.21387

Siegel, R. L., Miller, K. D., and Jemal, A. (2019). Cancer statistics, 2019. CA Canc. J. Clin. 69 (1), 7-34. doi:10.3322/caac.21551

Singla, M., and Bhattacharyya, S. (2017). Autophagy as a potential therapeutic target during epithelial to mesenchymal transition in renal cell carcinoma: an in vitro study. Biomed. Pharmacother. 94, 332-340. doi:10.1016/j.biopha.2017. 07.070

Su, Y., Lu, J., Chen, X., Liang, C., Luo, P., Qin, C., et al. (2019). Long non-coding RNA HOTTIP affects renal cell carcinoma progression by regulating autophagy via the PI3K/Akt/Atg13 signaling pathway. J. Canc. Res. Clin. Oncol. 145 (3), 573-588. doi:10.1007/s00432-018-2808-0

Sun, H., Wang, W., Che, Y., and Jiang, X. (2016). Fungal secondary metabolites rasfonin induces autophagy, apoptosis and necroptosis in renal cancer cell line. Mycology. 7 (2), 81-87. doi:10.1080/21501203.2016.1181114
Tang, J., Di, J., Cao, H., Bai, J., and Zheng, J. (2015). p53-mediated autophagic regulation: a prospective strategy for cancer therapy. Canc. Lett. 363 (2), 101-107. doi:10.1016/j.canlet.2015.04.014

Tanida, I., Minematsu-Ikeguchi, N., Ueno, T., and Kominami, E. (2005). Lysosomal turnover, but not a cellular level, of endogenous LC3 is a marker for autophagy. Autophagy. 1 (2), 84-91. doi:10.4161/auto.1.2.1697

Turcotte, S., Chan, D. A., Sutphin, P. D., Hay, M. P., Denny, W. A., and Giaccia, A. J. (2008). A molecule targeting VHL-deficient renal cell carcinoma that induces autophagy. Canc. Cell. 14 (1), 90-102. doi:10.1016/j.ccr.2008.06.004

Verine, J., Pluvinage, A., Bousquet, G., Lehmann-Che, J., de Bazelaire, C., Soufir, N., et al. (2010). Hereditary renal cancer syndromes: an update of a systematic review. Eur. Urol. 58 (5), 701-710. doi:10.1016/j.eururo.2010.08.031

Voss, M. H., Molina, A. M., and Motzer, R. J. (2011). mTOR inhibitors in advanced renal cell carcinoma. Hematol. Oncol. Clin. N. Am. 25 (4), 835-852. doi:10. 1016/j.hoc.2011.04.008

Wang, Z. L., Deng, Q., Chong, T., and Wang, Z. M. (2018). Autophagy suppresses the proliferation of renal carcinoma cell. Eur. Rev. Med. Pharmacol. Sci. 22 (2), 343-350. doi:10.26355/eurrev_201801_14178

White, E. (2016). Autophagy and p53. Cold Spring Harb. Perspect. Med. 6 (4), a026120. doi:10.1101/cshperspect.a026120

Wu, S. Y., Sung, P. J., Chang, Y. L., Pan, S. L., and Teng, C. M. (2015). Heteronemin, a spongean sesterterpene, induces cell apoptosis and autophagy in human renal carcinoma cells. Biomed. Res. Int. 2015, 738241. doi:10.1155/2015/738241

Yu, Z., Ma, J., Li, X., Liu, Y., Li, M., Wang, L., et al. (2018). Autophagy defects and related genetic variations in renal cell carcinoma with eosinophilic cytoplasmic inclusions. Sci. Rep. 8 (1), 9972. doi:10.1038/s41598-018-28369-y

Zhang, Q., Si, S., Schoen, S., Chen, J., Jin, X. B., and Wu, G. (2013). Suppression of autophagy enhances preferential toxicity of paclitaxel to folliculin-deficient renal cancer cells. J. Exp. Clin. Canc. Res. 32, 99. doi:10.1186/1756-9966-32-99

Conflict of Interest: The authors declare that the research was conducted in the absence of any commercial or financial relationships that could be construed as a potential conflict of interest.

Copyright (c) 2021 He and Tian. This is an open-access article distributed under the terms of the Creative Commons Attribution License (CC BY). The use, distribution or reproduction in other forums is permitted, provided the original author(s) and the copyright owner(s) are credited and that the original publication in this journal is cited, in accordance with accepted academic practice. No use, distribution or reproduction is permitted which does not comply with these terms. 